\title{
Activation of AhR with nuclear IKKa regulates cancer stem-like properties in the occurrence of radioresistance
}

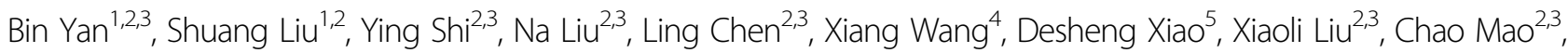

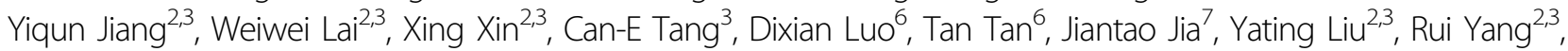 \\ Jun Huang ${ }^{8}$, Hu Zhou ${ }^{9}$, Yan Cheng ${ }^{10}$, Ya Cao ${ }^{2,3}$, Weishi Yu $^{11}$, Kathrin Muegge and Yongguang Tao $\mathbb{B}^{1,2,3,4}$
}

\begin{abstract}
Most cancer patients receive radiotherapy in the course of their disease and the occurrence of radioresistance is associated with poor prognosis. The molecular pathways that drive enhanced tumorigenic potential during the development of radioresistance are poorly understood. Here, we demonstrate that aryl hydrocarbon receptor (AhR) plays a vital role in the maintenance of cancer stem-like properties. AhR promotes the cancer stem-like phenotype and drives metastasis by directly targeting the promoters of 'stemness' genes, such as the ATP-binding cassette sub-family G member 2 (ABCG2) gene. Moreover, the radioresistant sublines display high levels of oncometabolites including aketoglutarate, and treatment of cancer cells with a-ketoglutarate enhances their stem-like properties in an AhR activation-dependent manner. IKKa directly activates stemness-related genes through an interaction with AhR as a bone fide chromatin modifier. Thus, AhR is functionally linked with cancer stem-like properties, and it drives tumorigenesis in the occurrence of radioresistance.
\end{abstract}

\section{Introduction}

The aryl hydrocarbon receptor (AhR), a ligand-operated transcription factor, is a xenosensor traditionally associated with xenobiotic metabolism ${ }^{1}$. AhR facilitates tumor progression, disease tolerance defense, intestinal immunity, and B-cell proliferation ${ }^{2-5}$. Interestingly, AhR influences the major stages of tumorigenesis, and studies of aggressive tumors and tumor cell lines have shown increased levels of AhR protein and constitutive nuclear

Correspondence: Shuang Liu (shuangliu2016@csu.edu.cn) or Yongguang Tao (taoyong@csu.edu.cn)

${ }^{1}$ Institute of Medical Sciences, Xiangya Hospital, Central South University, 87 Xiangya Road, Changsha 410008 Hunan, China

${ }^{2}$ Key Laboratory of Carcinogenesis and Cancer Invasion, Ministry of Education, Xiangya Hospital, Central South University, 87 Xiangya Road, Changsha 410008 Hunan, China

Full list of author information is available at the end of the article

These authors contributed equally: Bin Yan, Shuang Liu.

Edited by Q Chen localization in cancer tissue, whereas in normal tissues AhR is mainly inactive and resides in the cytoplasm ${ }^{6,7}$.

The activation of nuclear factor (NF)- $\kappa B$ leads to a protumorigenic inflammatory microenvironment, and the IkB-kinase (IKK) complex, which consists of two catalytic subunits, IKK $\alpha$ and IKK $\beta$, and a regulatory subunit, IKK $\gamma$, tightly regulates the NF- $\mathrm{KB}$ pathway, ${ }^{8,9}$. Whereas, in most malignancies, the classical IKK $\beta / \mathrm{IKK} \gamma$-dependent NF- $\mathrm{KB}$ activation controls key functions for tumor initiation, promotion, and progression in tumors ${ }^{10}$. The role of IKK $\alpha$ is more complex in noncanonical NF- $\mathrm{kB}$ pathway ${ }^{11,12}$. Depending on the type of malignancy, IKK $\alpha$ can provide both tumor-promoting and tumor-suppressive mechanisms that are in most instances cell autonomous ${ }^{13}$.

Radiotherapy, using ionizing radiation, is a commonly applied procedure for the treatment of cancers including lung cancer (LC) and nasopharyngeal carcinoma (NPC). Even though the technology of radiotherapy, including the

\section{(c) The Author(s) 2018}

(c) (i) Open Access This article is licensed under a Creative Commons Attribution 4.0 International License, which permits use, sharing, adaptation, distribution and reproduction c. in any medium or format, as long as you give appropriate credit to the original author(s) and the source, provide a link to the Creative Commons license, and indicate if changes were made. The images or other third party material in this article are included in the article's Creative Commons license, unless indicated otherwise in a credit line to the material. If material is not included in the article's Creative Commons license and your intended use is not permitted by statutory regulation or exceeds the permitted use, you will need to obtain permission directly from the copyright holder. To view a copy of this license, visit http://creativecommons.org/licenses/by/4.0/. 


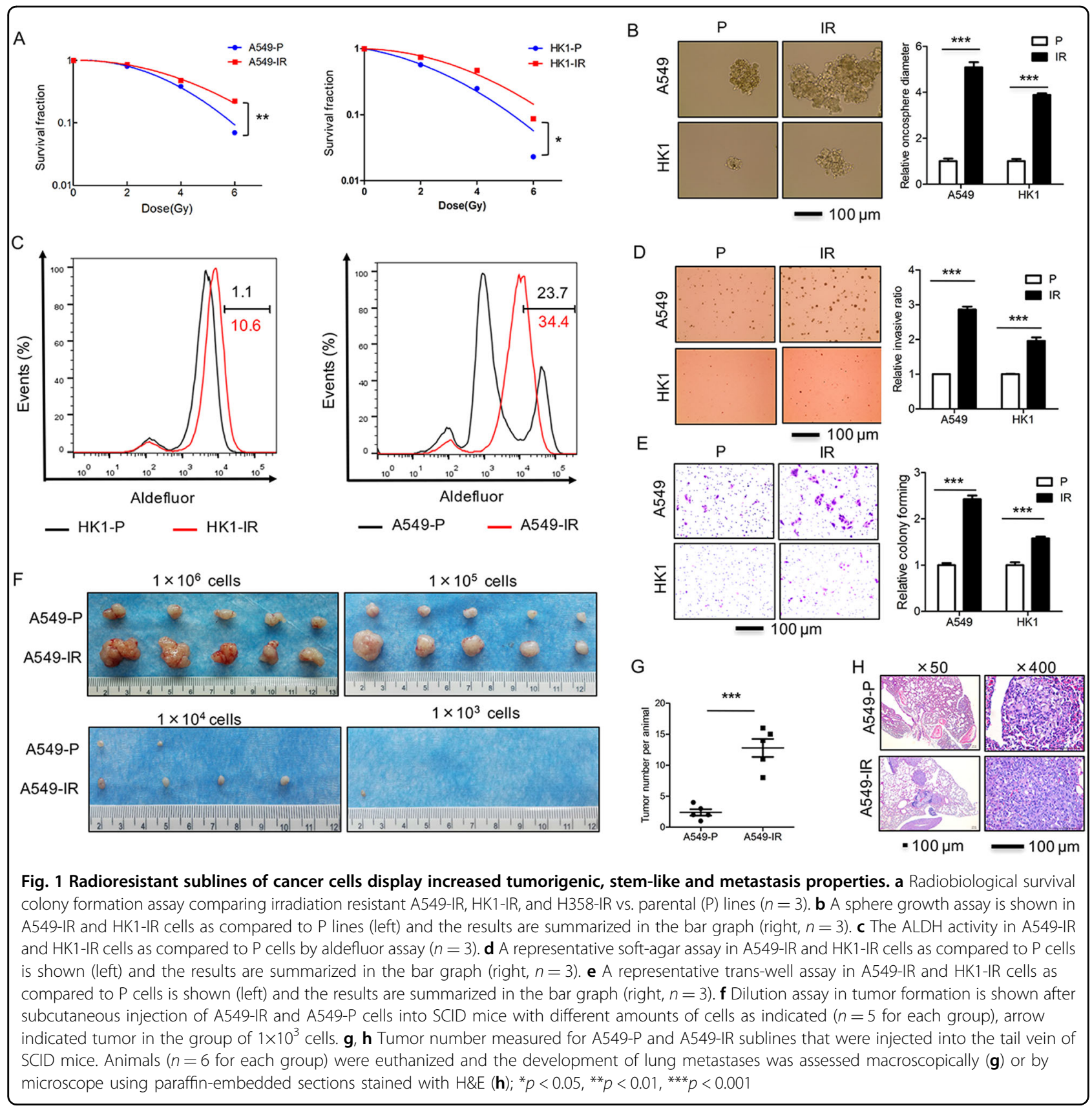

quality of the equipment and the precision of targeting, has greatly improved over the last decades, residual tumor tissues after irradiation and relapse due to radioresistant cancer cells remain a major challenge. The small radioresistant tumor subpopulation, known as "cancer stem cells" (CSCs), possesses specific molecular properties that protects it against radiation-induced damage and plays a critical role in tissue invasion and metastasis ${ }^{14-16}$. Several markers are known to characterize CSCs, including CD133, CD44, ATP-binding cassette sub-family G member 2 (ABCG2, also named as CD338), and epithelial cell adhesion molecule (Epcam, also named as CD326), stemness-related transcription factors Nanog, Octamer binding transcription factor 4 (Oct4), Krüppel-like factor 4 (KLF4), and aldehyde dehydrogenase (ALDH) activity $^{15,17-19}$. While several of these genes promote the stemness of CSCs, their exact roles in radioresistance have not been fully elucidated.

Accumulating evidence supports the existence of CSCs such as those derived from irradiation-resistant cells that possess the capacity to self-renew and to differentiate into bulk tumor cells ${ }^{20}$. In this study, we report that AhR is 
functionally linked with cancer stem-like properties, and it drives tumorigenesis in the occurrence of radioresistance.
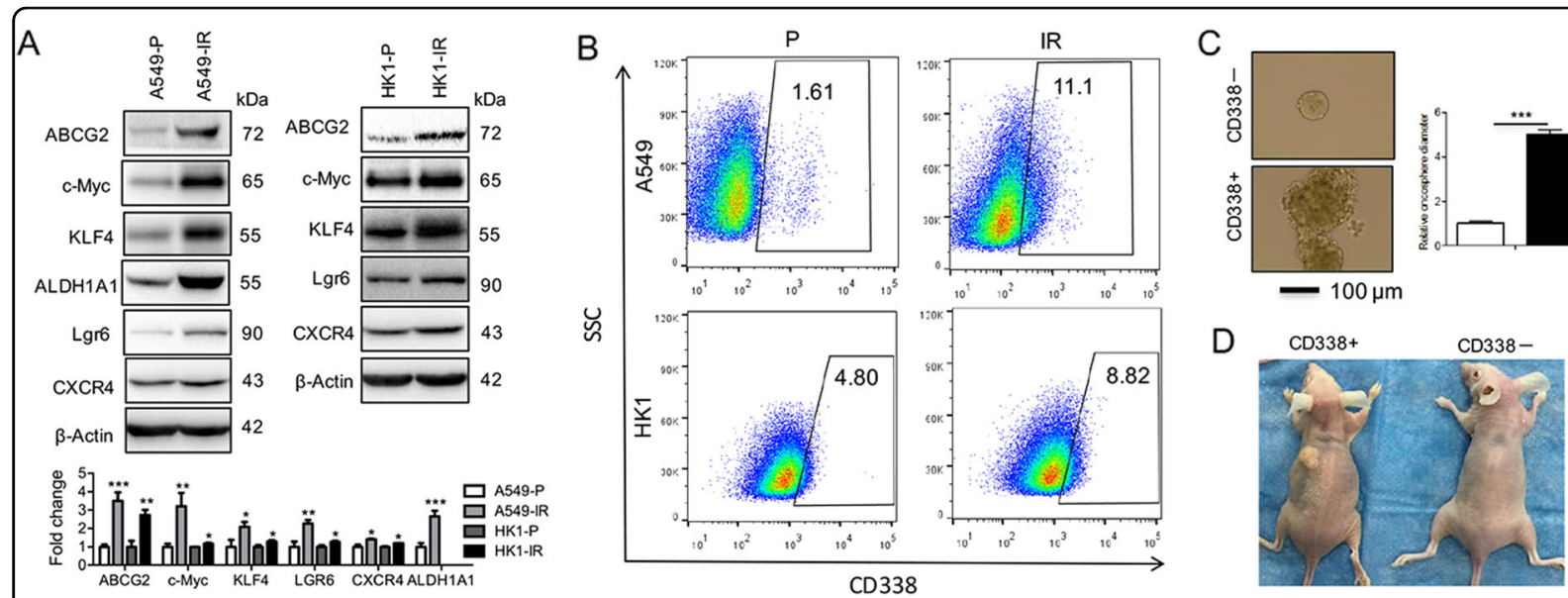

E
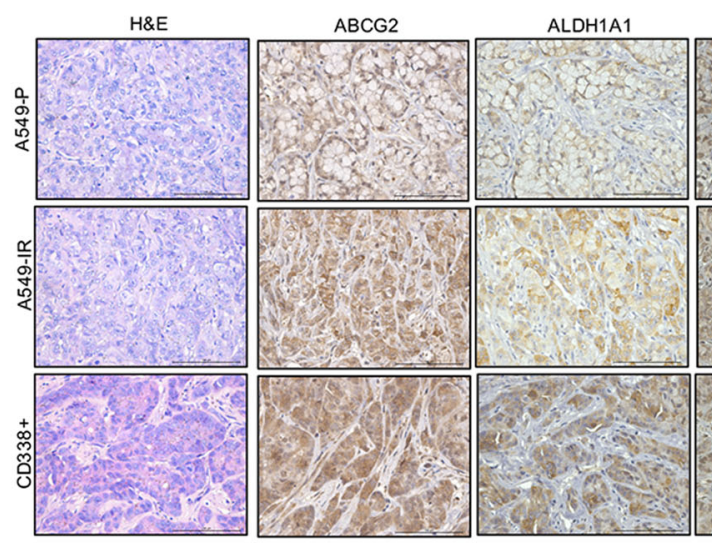

KLF4

Lgr6

CXCR4

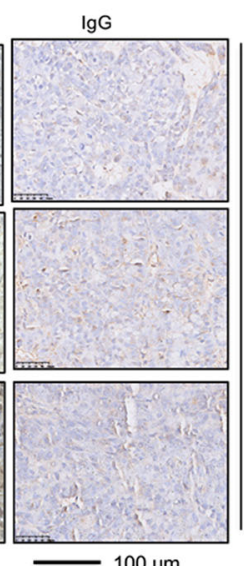

$\mathrm{F}$
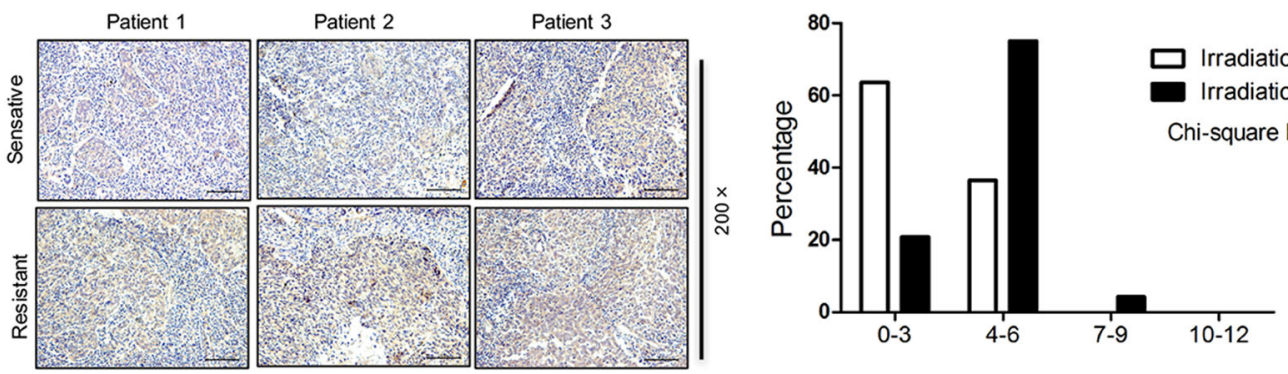

G
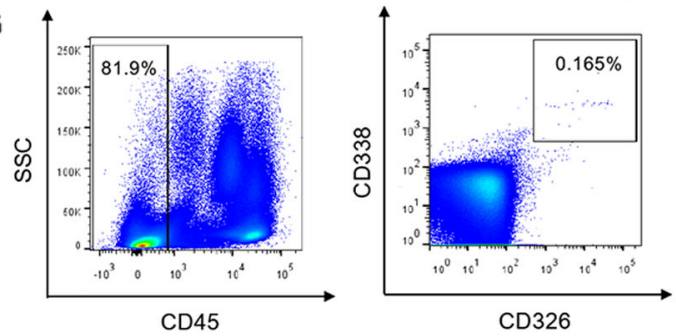

$\mathrm{H}$

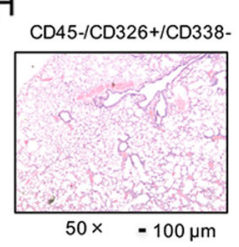

CD45-/CD326+/CD338+

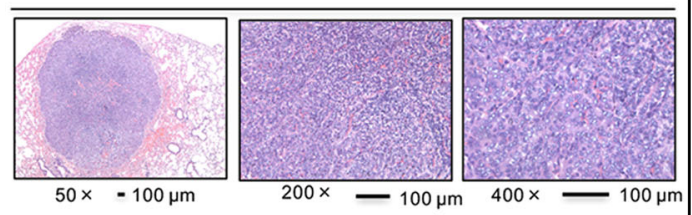

Fig. 2 (See legend on next page.) 
(see figure on previous page)

Fig. 2 The radioresistant sublines of cancer cells display elevated stem-like genes including ABCG2. a Western blot for detection of the expression of stemness-related genes as indicated in A549-P and A549-IR sublines (left), HK1-P and HK1-IR cells (right) $(n=3)$. The mean values of western blot quantification are shown at the bottom. $\mathbf{b}$ Representative images of flow cytometry analysis for detection of CD338-positive cells in A549-IR (up) and HK1-IR (bottom) sublines $(n=3)$. c Representative images of CD338-positive and -negative cells that were seeded in tumorspheric culture medium $(n=3)$. $\mathbf{d}$ Nude mice are shown after injection of CD338-positive and -negative cells from A549-IR cells. e H\&E staining (left) and immunohistochemical analysis to determine the expression of stemness-related genes as indicated in tumor samples generated in nude mice. $\mathbf{f}$ Immunohistochemical analysis of ABCG2 in three images of radiosensitive and radioresistant NPC tissues (left) and anti-ABCG2 staining intensity was quantified in three microscopic fields for each tissues section to determine the ABCG2 expression level (right). $\mathbf{g}$ Representative images of flow cytometry analysis for CD45-/CD326+/CD338+ cells derived from five lung cancer patients. $\mathbf{h}$ Animals ( $n=5$ for each group) were euthanized and the development of lung metastases derived from CD45-/CD326+/CD338+ cells from lung cancer patients was assessed by microscope using paraffin-embedded sections stained with $\mathrm{H \& E} ;{ }^{*} p<0.05,{ }^{* *} p<0.01,{ }^{* * *} p<0.001$

\section{Results}

Radioresistant sublines of cancer cells display increased tumorigenic, stem-like and metastasis properties

As a tool to identify markers of radioresistance in cancer cells, we used ionizing radiation resistant (IR) sublines generated from epithelial cancer cell lines HK1, A549, and H358. The first line is derived from the tissues of NPC, whereas the latter two are lung adenocarcinoma. Cell cultures were treated with multiple fractions of 4 Gy of Xrays to a total dose of more than $80 \mathrm{~Gy}$. The radiobiological clonogenic assay indicated enhanced survival in irradiation resistant (IR) sublines compared to the nonirradiated parental (P) cell lines. A significant increase in the survival of IR cells compared to P cells was observed at all given doses (Fig. 1a, and Supplementary Figure S1A). We observed that both A549-IR and HK1-IR cells exhibited more stem-like properties, such as the capacity of sphere growth (Fig. 1b) and aldefluor assay in ALDH activity (Fig. 1c), compared to P cells. Furthermore, all IR sublines in A549-IR, HK1-IR, and H358-IR cells showed greater survival in the soft-agar colony assay and enhanced in vitro invasion ability compared to P cell lines (Fig. 1d, e and Supplementary Figure S1B).

To investigate in vivo tumor formation, we injected the $\mathrm{P}$ and IR sublines subcutaneously into Balb/C immunedeficient mice $\left(2 \times 10^{6}\right.$ cells/mouse). After 2 months, we found significantly larger tumors from A549-IR cells as compared to the parental A54-P cells; moreover, limiting dilution assays were further performed to determine the frequency of radioresistant sublines in A549-IR and P lines from xenografts (Fig. 1f), significantly A549-IR efficiently formed large subcutaneous tumors. To further examine the properties of metastasis, we directly injected the tumor cells $\left(1 \times 10^{6}\right.$ cells/mouse $)$ into the tail vein of $\mathrm{Balb} / \mathrm{C} \mathrm{nu} / \mathrm{nu}$ mice. All A549-IR recipient mice (5/5) exhibited increased numbers of metastasis compared to mice that received the $\mathrm{P}$ cells (Fig. $1 \mathrm{~g}$, $\mathrm{h}$ and Supplementary Figure S1C-D). Taken together, our results demonstrate that the IR sublines show greater tumorigenic, stem-like and metastatic potential compared to their P lines.
The radioresistant sublines of cancer cells display elevated stem-like genes including ABCG2

Side populations (SP) in cells may exhibit stem cell-like characteristics. Flow cytometry analysis indicated that the percentage of SP cells was greater in HK1-IR cells (10.6\%) compared to P cells (1.57\%) (Supplementary Figure S2A). IR cells in A549 and H358 expressed elevated mRNA of genes associated with a stem-like phenotype including ABCG2, c-Myc, KLF4, ALDH1A1, Lgr6, and CXCR4 (Supplementary Figure S2B). Moreover, the protein level of ABCG2, c-Myc, KLF4, ALDH1A1, Lgr6, and CXCR4 was elevated in A549-IR cells as compared to P cells (left panel of Fig. 2a), whereas the protein level of ABCG2, cMyc, KLF4, Lgr6, and CXCR4 was elevated in HK1-IR cells as compared to P cells (right panel of Fig. 2a), similar findings were seen in H358-IR cells compared to P cells (Supplementary Figure S2C). In addition, the percentage of CD338-positive cells, as measured by flow cytometry analysis, was higher in A549-IR and HK1-IR cells as in their respective $\mathrm{P}$ lines (Fig. $2 \mathrm{~b}$ ). We observed that CD338-positive cells exhibited more stem-like properties in the capacity for sphere growth (Fig. 2c). As few as 1000 CD338-positive A549-IR cells that were subcutaneously injected into Balb/C immune-deficient mice resulted in tumor formation $(2 / 5)$, compared to no tumor formation $(0 / 5)$ after injection of CD338 negative cells (A presentative image is shown in Fig. 2d). The expression of CSC markers ABCG2, ALDH1A1, KLF4, and LGR6 was readily detected in biopsies of xenograft tumors after injection of IR cells and after injection of CD338-positive cells into mice compared to P cells (Fig. 2e). Moreover, we examined ABCG2 protein levels in NPC tissues by immunohistochemistry analysis; ABCG2 expression was significantly increased in biopsies of radioresistant NPCs as compared to radiosensitive NPC tissues (Fig. 2f). Finally, we sorted ABCG2 cells derived from five patients with lung adenocarcinoma after permission. To get more specific ABCG2-positive tumor-initiating cells from LC patients, we sorted CD45-/CD326+/CD338+ cells from $10.0 \mathrm{ml}$ of blood cells from LC patients (Fig. 2g); then we injected the cells into the tail vein of $\mathrm{Balb} / \mathrm{C} \mathrm{nu/nu}$ mice 
for 4 months. Interestingly, all CD45-/CD326+/CD338+ cells recipient mice (5/5) exhibited tumor formation in the lung compared to mice that received the CD45 -/CD326-/CD338- cells (0/5) (Fig. 2h and Supplementary Figure S2D). Taken together, our data show an association of radiation resistance with elevated stem-like gene signature and enhanced tumorigenic potential.

\section{Stem-like genes are directly contributed by AhR in radioresistant sublines}

To understand more about the regulation of stemness genes, we searched for a common molecular pathway in IR sublines. The UCSC genome browser (www.genome. ucsc.edu) reveals that the promoter regions of stemness marker genes contain AhR binding sites. ChIP analysis demonstrated that $\mathrm{AhR}$ was recruited to the promoters of several stemness genes including ABCG2, c-Myc, KLF4, ALDH1A1, and Lgr6 in P cell lines A549 and $\mathrm{HK} 1$, suggesting a possible regulatory role of this transcription factor in the expression of stemness genes (Fig. 3a). Notably, the enrichment of AhR at the promoter regions was increased in radioresistant cells lines A549-IR and HK1-IR that showed also higher expression of those stemness-related genes (Fig. 3a). Interestingly, not only $A h R$ promoter occupancy but also the total AhR protein expression levels were consistently elevated in radioresistant sublines compared to $P$ lines (Fig. 3b), whereas the mRNA level of AhR remained the same in radioresistant sublines compared to $\mathrm{P}$ lines (data not shown). Moreover, AhR expression was increased in A549-IR and CD338-positive A549-IR and CD338+ biopsies from xenograft tumors (Supplementary Figure S3A); meanwhile, the mRNA level of CYP1A1, a bona fide target gene of AhR, was increased in A549-IR and HK1-IR cells (Fig. 3c), indicating that higher activation of AhR signaling pathway exists in radioresistant sublines.

Since AhR can be found in the cytoplasm and in the nucleus, we confirmed that the nuclear fraction of AhR was indeed increased in A549-IR and HK1-IR cells as an active form (Fig. 3d). Immunofluorescence staining (Supplementary Figure S3B) together with the high content imaging system (Supplementary Figure S3C-E) confirmed that the nuclear localization of AhR increased in the HK1-IR and A549-IR sublines as compared to P cells. We examined the AhR protein levels in control (NP) and NPC tissues by immunohistochemistry analysis. While low levels of AhR protein were detectable in NP tissue, AhR was greatly increased in NPCs, and the degree of expression was associated with expression of the Epstein-Barr virus (EBV)-encoded RNA (Supplementary Figure S4A-B). Furthermore, AhR expression was significantly increased in biopsies of radioresistant NPCs as compared to radiosensitive NPC tissues (Fig. 3e). Also, the expression of AhR protein was elevated in NPC tissues of patients with metastasis compared to those with nonmetastasis (Supplementary Figure S4C, D).

\section{Knockdown of AhR decreases the stemness signature}

To validate the physiological role of AhR in the radioresistant sublines, we used four different AhR-targeting shRNAs to knock down AhR expression and observed that shAhR-2 consistently achieved over 95\% knockdown efficiency (Supplementary Figure S5A). Therefore, unless specified otherwise, shAhR-2 was used in the subsequent studies. As expect, knockdown of AhR decreased the expression of CYP1A1 and several stemness signature genes including ABCG2, c-Myc, KLF4, Lgr6, ALDH1A1, and CXCR4 at mRNA level in A549-IR and HK1-IR cells (Supplementary Figure S5B). Obviously, the knockdown of AhR decreased the protein level of stem-like genes including c-Myc, KLF4, LGR6, and CXCR4 in A549-IR and HK1-IR cells (Fig. 4a). FACS assay showed that knockdown of AhR lead to a decrease of CD338+ expression in A549-IR and HK1-IR cells (Fig. 4b). In the aldefluor assay, ALDH activity decreased after depletion of AhR in A549-IR and HK1-IR cells (Fig. 4c); A549-IR and HK1-IR cells depleted of AhR exhibited less stem-like properties in sphere growth (Fig. 4d), indicating that AhR contributes to stem-like properties in the radiation sublines.

Moreover, knockdown of AhR resulted in significantly reduced growth of A549-IR and HK1-IR cells as well as P cells in culture (Supplementary Figure S5C), and impaired the colony formation of HK1-P, HK1-IR and A549-IR cells in a plate colony formation assay (Supplementary Figure S5D-E). Furthermore, knockdown of AhR in A549IR cells led to a decreased activity for colony formation in a soft-agar assay (Fig. 4e) and invasion assay (Fig. 4f). Finally, knockdown of AhR resulted in significantly reduced tumor growth and malignant characteristics of A549-IR cells in nude mice (Fig. 4g and Supplementary Figure S5F); the expression of stemness signature genes including ABCG2, c-Myc, KLF4, Lgr6, ALDH1A1, and CXCR4 were readily decreased in biopsies of xenograft tumors after knockdown of AhR in A549-IR cells (Fig. 4h). Taken together, these findings indicate a critical role of AhR in the maintenance of stem-like properties of the IR sublines.

\section{Activation of AhR signaling pathway is linked to stem-like properties in radioresistant sublines}

Given the link between reduced AhR expression and decreased stem-like properties, we assessed whether increased AhR activation might inversely modulate the process. When A549-IR and H358-IR cells were treated with an AhR agonist and antagonist, we found that CYP1A1 mRNA levels were modulated after treatment 


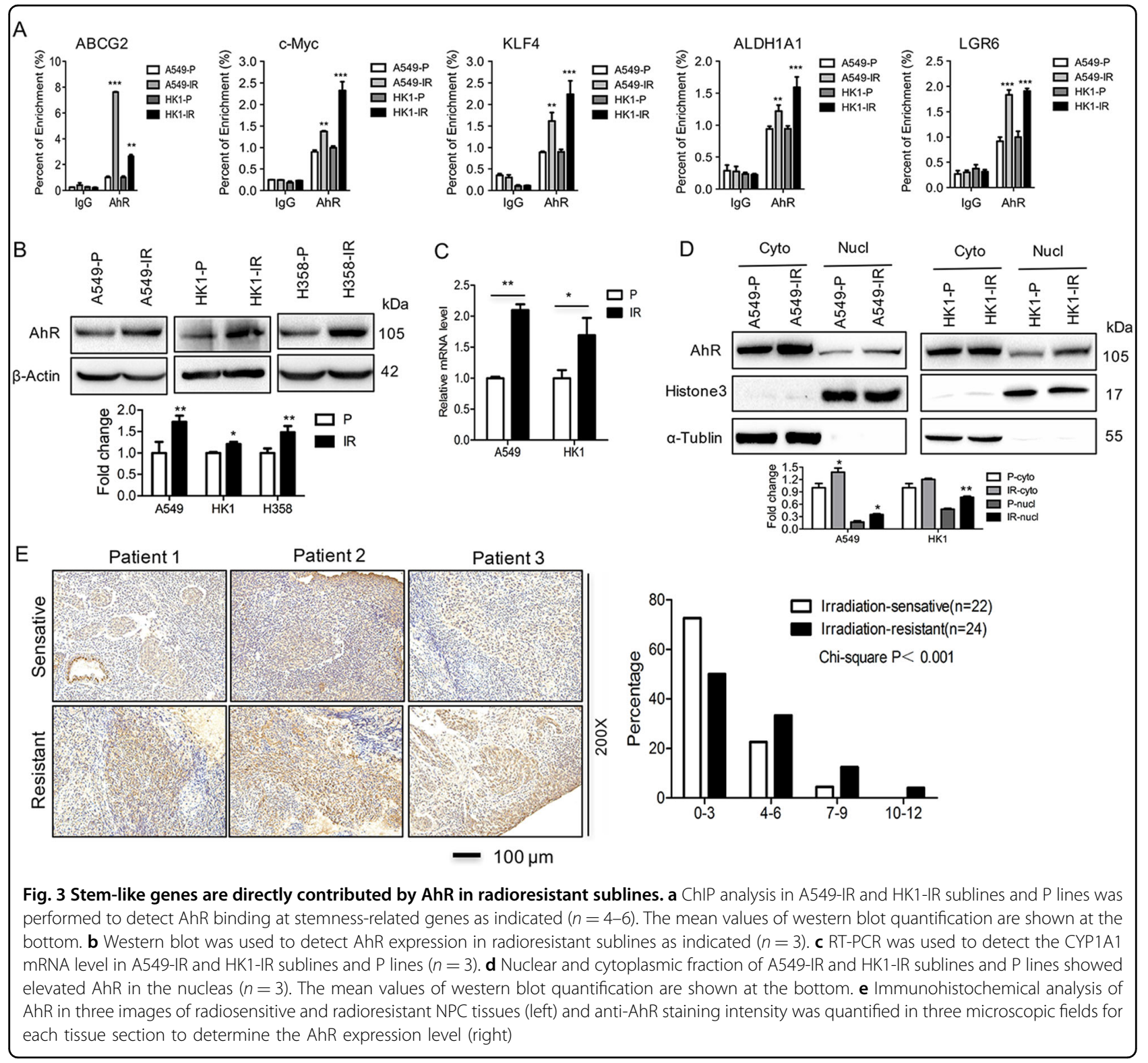

with the AhR agonist in A549-IR and H358-IR cells and the P cells (Supplementary Figure S6A-B), treatment with the AhR agonist also elevated ABCG2 mRNA (Supplementary Figure S6A-B). Furthermore, treatment of A549IR cells with an AhR agonist elevated the percent of CD338+ cells, whereas the AhR antagonist decreased the number of CD338+ cells (Fig. 5a). Treatment with the AhR agonist elevated the ABCG2 protein levels in HK1 and H358 P cells as well as H358-IR cells (Supplementary Figure $\mathrm{S6C}-\mathrm{D})$, moreover, the $\mathrm{AhR}$ agonist elevated the protein levels of ABCG2, c-Myc, and KLF4 in A549 P cells (Supplementary Figure S6E). The soft-agar assay showed that AhR agonist, not AhR antagonist, treatment increased colony formation in A549-IR cells (Supplementary Figure S6F). Moreover, stemness-related genes including ABCG2, c-Myc, and KLF4 were not induced by AhR agonist after depletion of AhR in both HK1-IR (Supplementary Figure S6G) and A549-IR cells (Supplementary Figure $\mathrm{S} 6 \mathrm{H}$ ) at mRNA levels, indicating that the induction of stemness-related genes is dependent on the activation of AhR signaling pathway.

The AhR pathway can cross talk with other major signaling pathways that might be modulated by oncometabolites that are critical in cancer progression ${ }^{21-23}$. We found elevated oncometabolite levels including 2hydroxyglutarate (2-HG), $\alpha$-ketoglutarate $(\alpha-K G)$, citrate, fumarate, malate, and succinate in A549-IR and HK1-IR cell sublines compared to P lines (Fig. 5b, c). Furthermore, after addition of $\alpha-K G$, we observed that the colony numbers increased in A549-IR cells, whereas 


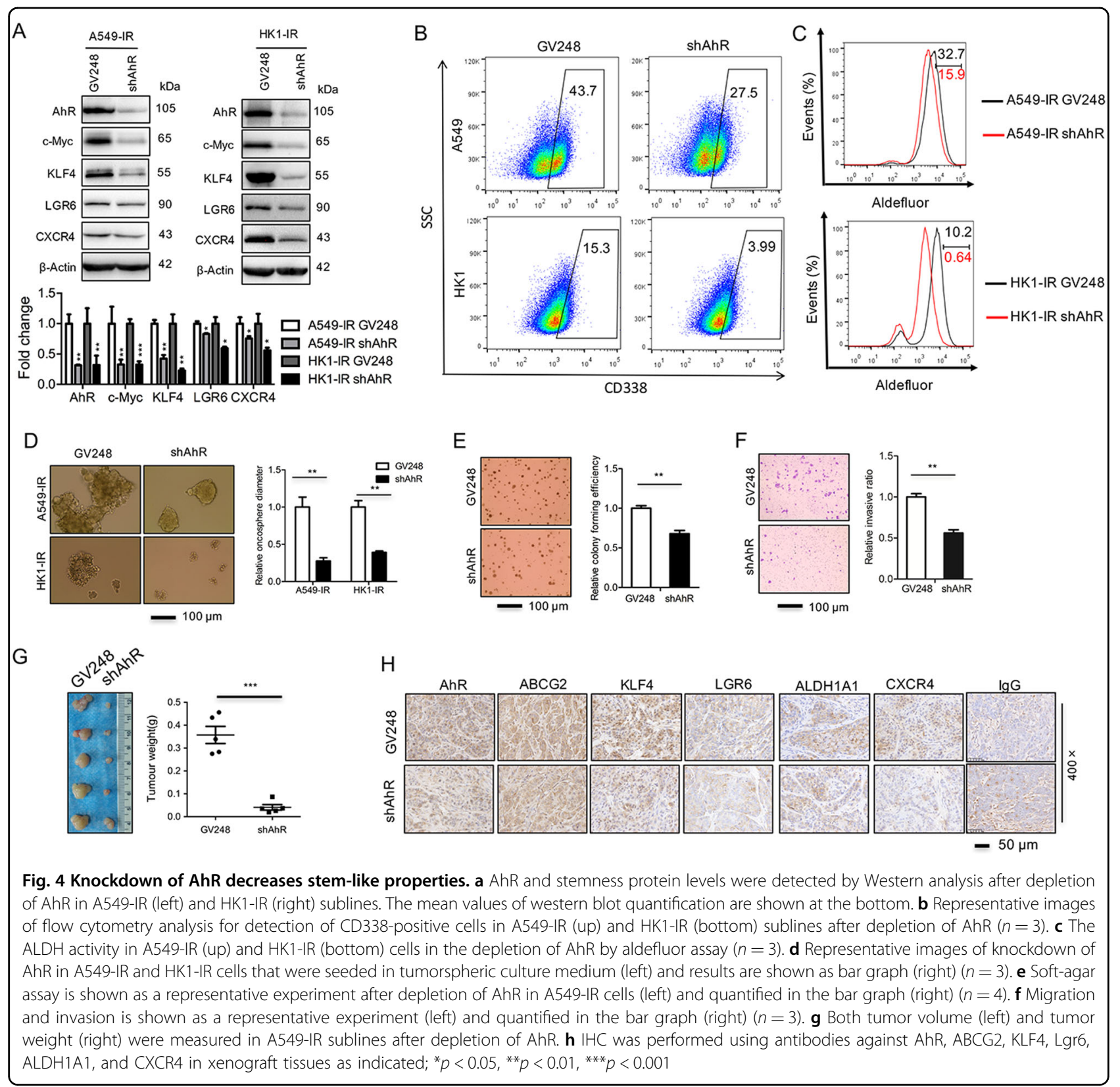

addition of succinate did not change the colony number in the soft-agar assay (Fig. 5d). Interestingly, the addition of $\alpha-K G$, not succinate, triggered the activation of AhR through detection of CYP1A1 (Supplementary Figure S7B). Furthermore, addition of $\alpha-K G$, but not succinate, induced an increase of ABCG2 mRNA and protein levels in A549-IR and P lines (Fig. 5e and Supplementary Figure S7C), and flow cytometry showed that $\alpha-K G$ increased the percent of CD338+ cells from 3.13 to 4.9 (Fig. 5f), indicating that $\alpha$-KG might induce cancer stem-like properties through triggering AhR signaling pathway. As AhR is localized in the nucleus as an active form, we found that the nuclear fraction of
AhR was indeed increased in A549-P and HK1-P cells with the addition of $\alpha-K G$ (Fig. $5 \mathrm{~g}$ ). Using ChIP analysis, we observed increases in AhR occupancy at the promoter region of several stemness-related genes including ABCG2, c-Myc, KLF4, ALDH1A1, and LGR6 after the addition of $\alpha-K G$ (Fig. $5 \mathrm{~h}$ ). Interestingly, addition of $\alpha-K G$ increased the mRNA expression of CYP1A1, ABCG2, c-Myc, and KLF4 in A549-P cells, this increase was abolished after depletion of AhR using shRNA knockdown (Fig. 5i), indicating a functional link between $\alpha-K G$ and AhR in a dependent manner. In summary, activation of the AhR is linked with stem-like property. 


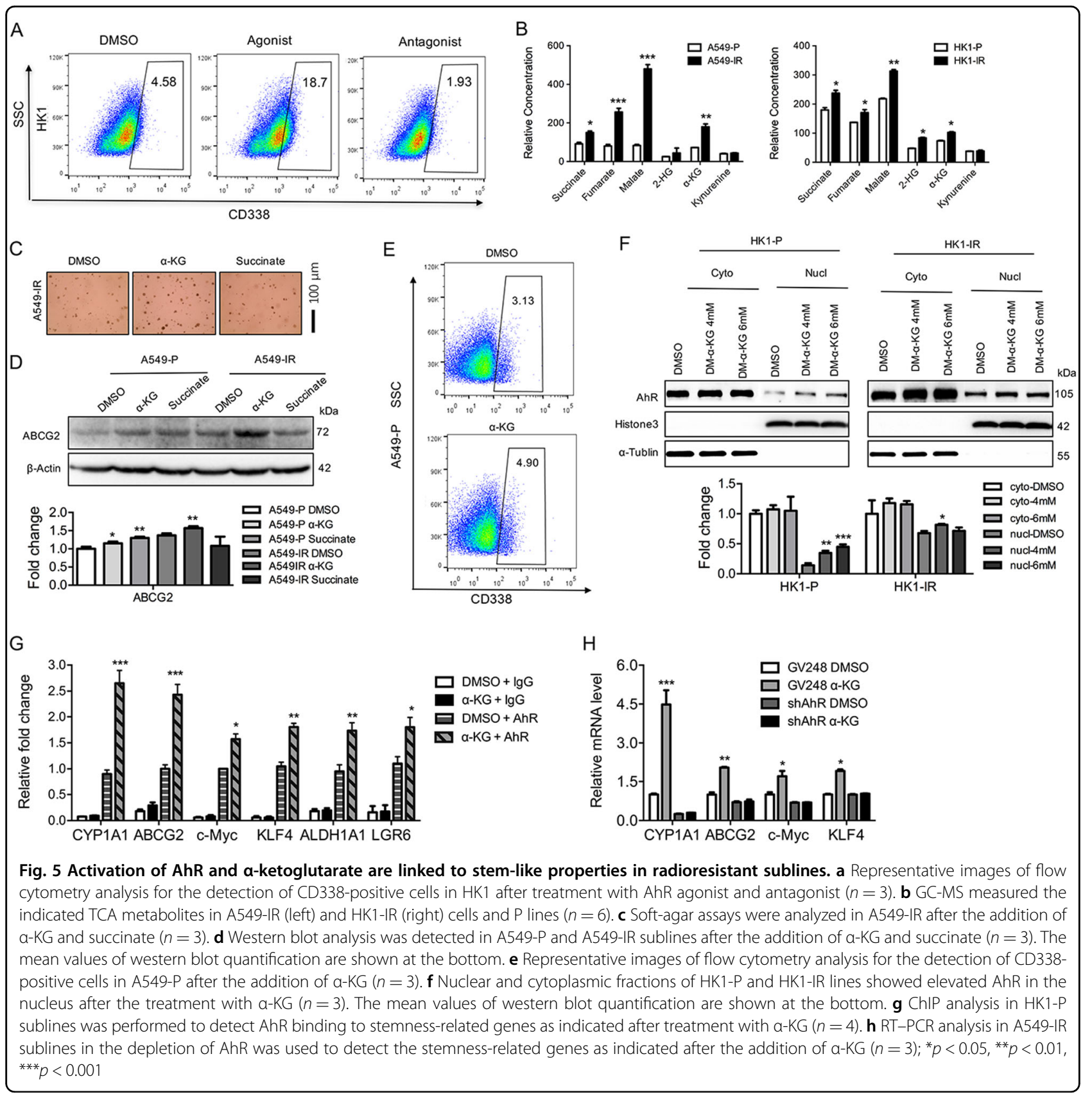

\section{Nuclear IKKa involves in the regulation of AhR in stemness-related genes}

We have recently shown that IKK $\alpha$ can localize to the nucleus and directly bind to the target gene's promoters ${ }^{13,24,25}$. Since we observed that the nuclear levels of IKK $\alpha$ increased in A549-IR and HK1-IR cells compared to P cells (Fig. 6a), we tested whether IKK $\alpha$ and AhR may form an intact complex. Using immunofluorescence assay, we found that IKK $\alpha$ colocalized with AhR in the nucleus (Fig. 6b); we further found evidence of IKK $\alpha$ and AhR interaction in IR sublines and P lines using co-immunoprecipitation assay, whereas the intact complex of IKK $\alpha$ and AhR formed in A549-IR and HK1IR cells as compared to P cells (Fig. 6c). On the basis of higher nuclear localization of IKK $\alpha$ in radioresistant sublines, ChIP analysis demonstrated an increased enrichment of IKK $\alpha$ at the promoter of stemness-related genes including ABCG2, c-Myc, KLF4, Lgr6, and ALDH1A1 in A549-IR and HK1-IR cell sublines compared to $\mathrm{P}$ lines (Fig. $6 \mathrm{~d}$ ), indicating that IKK $\alpha$ directly involves in the regulation of stemness genes. Furthermore, in order to investigate whether the binding of IKK $\alpha$ at the promoter of stemness-related genes is dependent of AhR, we performed ChIP analysis and 


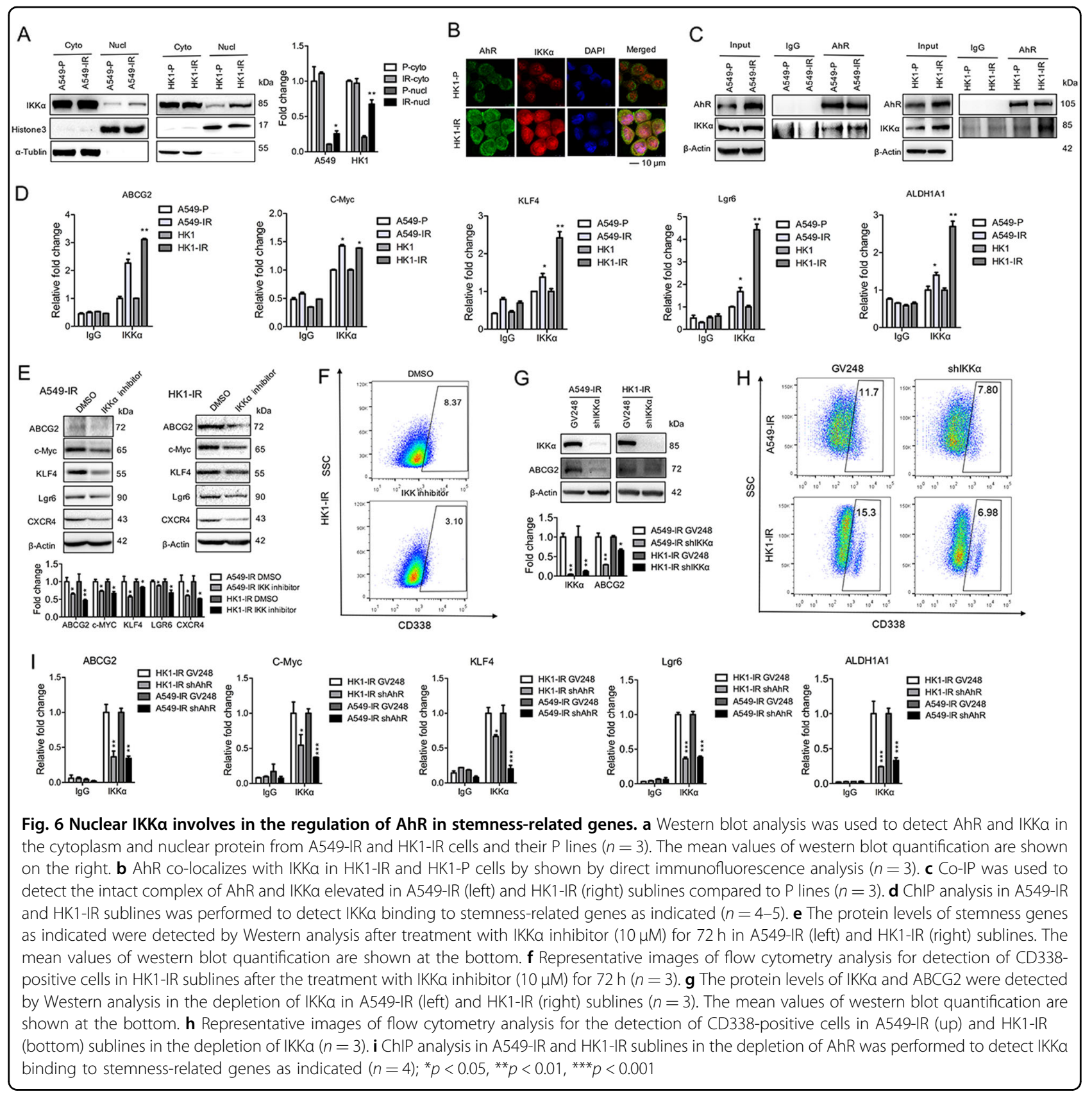

found that the enrichment of IKK $\alpha$ decreased at the promoter of stemness-related genes in HK1-IR and A549-IR cells after depletion of AhR (Fig. 6e), indicating that AhR is essential for IKK $\alpha$ recruitment.

To further confirm the critical role of IKK $\alpha$ in the regulation of stemness genes, we first treated A549-IR and HK1-IR cells with IKK $\alpha$ inhibitor, IKK inhibitor XII, a selective inhibitor of IKK $\alpha$ activity. Figure $6 f$ showed that the inhibitor of IKK $\alpha$ reduced the protein levels of stemnes genes including ABCG2, c-Myc, KLF4, Lgr6, and CXCR4. FACS assay showed that the IKK $\alpha$ inhibitor lead to a decrease of CD338+ expression in HK1-IR cells compared to P lines (Fig. 6g). Moreover, depletion of IKK $\alpha$ also decreased ABCG2 expression in A549-IR and HK1-IR cells (Fig. 6h); also, knockdown of IKK $\alpha$ decreased the percent of CD338 positive in A549-IR and HK1-IR cells (Fig. 6i). Taken together, these data suggest a critical role of IKKo in the regulation of stemness-related genes in radioresistant cells through an interaction with AhR.

Based on our findings, we propose a model (Fig. 7). In this model, AhR acts as a core component of radioresistance involved in tumorigenic, stem-like and metastatic properties. 


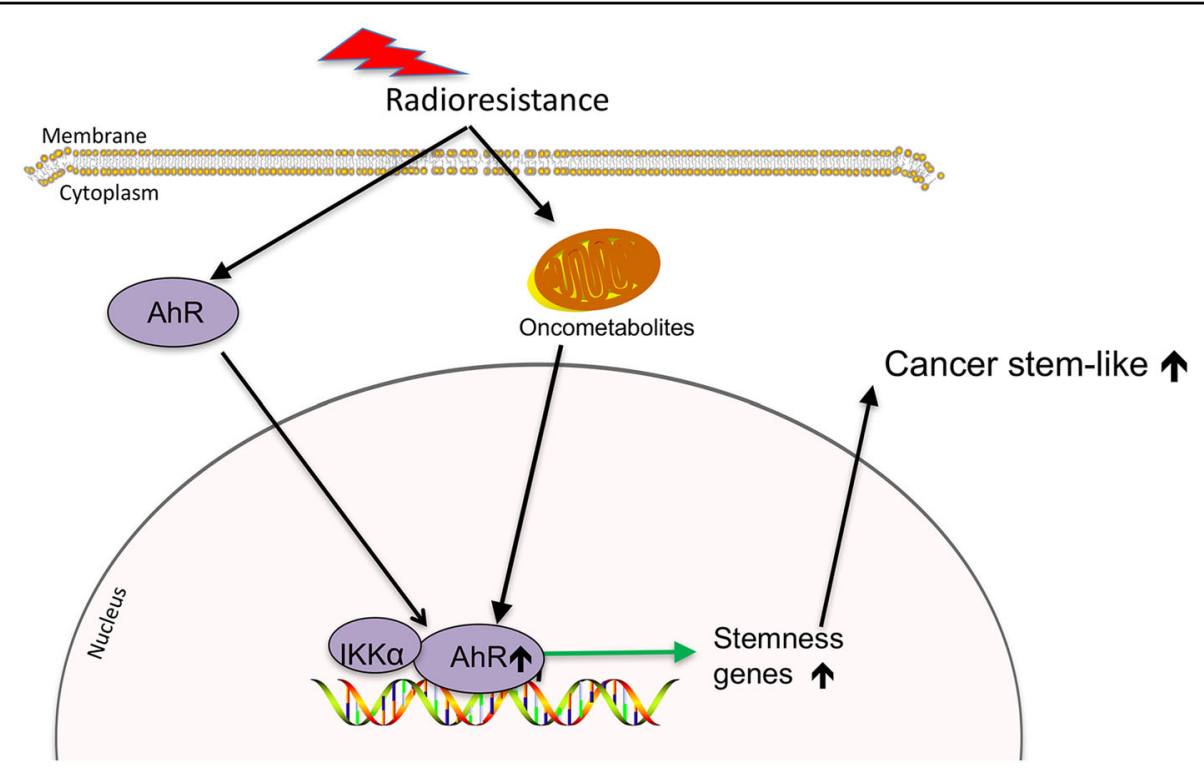

Fig. 7 The schematic model of AhR in tumorigenic, stem-like, metastasis properties in the occurrence of radioresistance. AhR induces stemlike properties by directly targeting the promoters of stemness-related genes; the intact complex of IKKa and AhR also directly activates the stemness-related genes. Increased generation of oncometabolites in radioresistant sublines, in turn, induces stem-like signature gene expression in an AhR-dependent manner

\section{Discussion}

Approximately $50 \%$ of all cancer patients receive radiotherapy, and it is worth mentioning that about $20 \%$ of those patients develop local recurrence which has been linked to the acquisition of radioresistance ${ }^{14}$. Several tumor-promoting genes such as CXCR4, LSH, MMP9, and hypoxia-inducible factor (HIF)- $1 \alpha$ are thought to contribute to radioresistance, and their elevated expression is consistent with their proposed role in cancer progression, metastasis, and radiotherapy ${ }^{24,26-30}$.

AhR, a widely expressed nuclear receptor that senses environmental stimuli and modulates target gene expression, plays a critical role in breast CSCs etc ${ }^{31,32}$. AhR influences the major stages of tumorigenesis and chemoresistance $^{33-35}$, and studies of aggressive tumors and tumor cell lines have shown increased levels of AhR protein and constitutive nuclear localization in cancer tissue, whereas in normal tissues AhR is mainly inactive and resides in the cytoplasm ${ }^{6,7}$. Here we found elevated AhR level in the nucleus linked with radioresistance in LC and NPC cells, indicating that activation of AhR contributes to cancer progression, stem-like properties, and radioresistance. Nuclear IKK $\alpha$ transcriptionally upregulates several miRNAs, contributing to chemoresistance ${ }^{36}$. Recently, mixed lineage kinase 4 binds and phosphorylates IKK $\alpha$, leading to activation of NF- $\mathrm{KB}$ signaling in glioma stem cells ${ }^{37}$. Here we show that nuclear IKK $\alpha$ targets the promoters of stem-like genes as a partner of AhR, contributing to radioresistance, indicating a critical role of nuclear IKK $\alpha$ in cancer progression and radioresistance. Moreover, we found that the protein level of AhR, not mRNA level, increased in radioresistant sublines.

There is an opposed effect of IKK $\alpha$ in several types of cancer in the process of carcinogenesis. For example, inhibition of IKK $\alpha$ prolongs survival and suppresses the occurrence of metastatic diseases in models of mammary, prostate, and colorectal cancers $^{38-42}$, whereas, IKK $\alpha$ controls metastasis in breast and prostate cancers and is required for mammary tumorigenesis through expansion of tumor-initiating cells ${ }^{39-41}$. In contrast, IKK $\alpha$ acts as a tumor suppressor in models of skin or lung (Squamous cell carcinoma) SCC, while loss of IKK $\alpha$ enhances susceptibility to carcinogen-induced SCC in the skin and leads to the development of spontaneous lung $\mathrm{SCC}^{43,44}$. Recently, we show that IKK $\alpha$ is diversely expressed in keratinizing and non-keratinizing carcinomas in the same type of cancer $^{13}$. We also provide evidence that IKK $\alpha$ is absent in SCC of the skin while it is expressed in (Basalcell carcinoma) $\mathrm{BCC}$, indicating the opposed roles of IKK $\alpha$ in skin cancer between SCCs and BCCs, which originate from both keratinocyte tumors ${ }^{25}$. Moreover, SCC development is generally associated with cell dedifferentiation that IKK $\alpha$ is reversely involved in the process. Here we show the critical role of IKK $\alpha$ in the regulation of stemness-related genes in radioresistant cells through an interaction with AhR.

Epithelial-mesenchymal transition (EMT) is a key factor of cancer progression, metastasis, and self-renewal of $\mathrm{CSCs}^{45-47}$. TGF- $\beta$ tumor suppression functions through 
an EMT-mediated disruption of a lineage-specific transcriptional network ${ }^{48}$. Interestingly, EMT is not required for metastasis but contributes to chemoresistance ${ }^{49,50}$. However, we did not find the classical features of EMT and (mesenchymal-epithelial transition) MET in the stem-like radioresistant cells, but a hybrid EMT/MET state (data not shown). This EMT/MET hybrid state is thought to be bidirectional displaying a gradient of partial states toward either extreme EMT or extreme MET, and further supports the importance for metastasis and metastasis-initiating cells as well as circulating tumor cells in the epithelial and mesenchymal composition ${ }^{51-54}$, and it also supports that an intermediate EMT state may make cells more prone to exhibiting stem-like properties ${ }^{54}$. Our findings indicate that stemness seems to be better manifested at intermediate epitheloid states, consistent with those observed in cultured mammospheres. In particular, tumor-associated fibroblasts may be responsible for inducing EMT by secreting TGF- $\beta$ that can stimulate proliferation and lead to expansion of the preexisting CSC pool $^{55,56}$, even though we did not find that TGF- $\beta$ changed in radioresistant cells.

The AhR pathway can cross talk with other major signaling pathways that might be modulated by oncometabolites that are critical in cancer progression ${ }^{21-23}$. Diverse metabolites serve as cofactors or substrates for enzymes that are involved in the deposition or exchange of epigenetic marks, driving a metabolite-driven pathway of gene regulation as well as distinct cancer tissue types ${ }^{24,57-59}$. Also, organ-specific differences were observed in the metabolite levels of the TCA cycle and other intermediates $^{58,60}$. 2-HG is a competitive inhibitor of $\alpha$-KGdependent dioxygenases in gliomas and hematological malignancies that carry mutations of isocitrate dehydrogenase genes (IDH1 and IDH2) ${ }^{61,62}$. However, we did not find mutations of IDH1 and IDH2 genes and accumulation of 2-HG in NPC and LC (data not shown). We found that the level of kynurenine, which is involved in tryptophan catabolism, remained the same in radioresistant sublines compared to $\mathrm{P}$ sublines, indicating that kynurenine did not further activate AhR signaling pathway, but increased the generation of $\alpha-K G$ oncometabolite in radioresistant sublines, and in turn induced stemlike signature gene expression by AhR in a dependent manner. Here, we report that $\alpha-K G$ can activate the AhR signaling pathway leading to elevated stemness gene expression to influence the pluripotency state, and we provide evidence of a direct link between cellular metabolism, AhR signaling, and radioresistance associated with stem-like property.

Taken together, we elucidated the crucial role of AhR in the maintenance of radioresistance and stem-like property involving in direct activation of self-renewal via stemness genes, leading to metabolic reprogramming. Our data showed that stemness reprogramming controlled by metabolism can modulate radioresistance and tumor progression.

\section{Material and methods}

Cell culture, antibodies, plasmids, siRNAs, and chemicals

Lung adenocarcinoma cell lines A549-P/H358-P and its irradiation-resistant cell lines A549-IR/H358-IR were provided by Xinming Deng (Emory University School of Medicine and Winship Cancer Institute of Emory University, Atlanta, USA). A549-P/IR cell lines were maintained in DMEM/F12 and RPMI 1640 medium, respectively, which were supplemented with $10 \%$ fetal bovine serum (FBS). NPC cell line HK1 was used to establish ionizing radiation resistant cell line (HK1-IR), as described $^{63}$. Briefly, HK1 cells were serially irradiated with 4 Gy of X-rays to a final dose of 80 Gy using XRAD320 (Precision X-ray, Inc., North Branford, CT). HK1-P/IR cell lines were maintained in RPMI 1640 medium. All cell lines tested negative for mycoplasma contamination and were maintained at $37{ }^{\circ} \mathrm{C}$ with $5 \% \mathrm{CO}_{2}$. All cell lines were authenticated by short tandem repeat profiling and were passaged less than ten times after initial revival from frozen stocks.

Primary antibodies as indicated for Western analysis were purchased from Cell Signaling (Danvers, MA). Primary antibodies for AhR and $\beta$-actin were purchased from Sigma-Aldrich (St. Louis, MO). GV248-shAhR lentivirus plasmids were purchased from Genechem (www. genechem.com.cn). RNAi sequences used in these studies are provided (shAhR-1: CACAACAATATAATGTCTT; shAhR-2: TTCTTTGATGTTGCATTAA; shAhR-3: AATGATTAAGACTGGAGAA; shAhR-4: ATAATAACTCCTCAGACAT). The chemicals, citrate, $\alpha-K G$, 2-HG, fumarate, malate, succinate, oxalacetic acid, 2isopropylmalic acid, methanol, N,O-bis(trimethylsilyl) trifluoroacetamide, and trimethylcholorosilane and its dimethyl products were purchased from Sigma-Aldrich (St. Louis, MO). The chemicals AhR agonist V, VAF347 (Cat\# 182690), and AhR antagonist (Cat\# 182705) were purchased from Calbiochem.

\section{Western blot analysis and co-immunoprecipitation (Co-IP) assay}

Details of the western blot analysis and Co-IP assay were described previously ${ }^{24,29}$. A detailed procedure is presented in the Supplementary Material and Methods section. The following list of antibodies was used for western blot detection: AhR and IKK $\alpha$ antibodies.

\section{Quantitative real-time PCR}

Cells were harvested with Trizol (Invitrogen). cDNAs were synthesized with SuperScript III (Invitrogen) according to the manufacturer's protocol. Real-time PCR 
analysis was performed using the Applied Biosystems 7500 Real-Time PCR System, according to the manufacturer's instructions. The reactions were performed in triplicates for three independent experiments: the results were normalized to $\beta$-actin. For qPCR, an SYBR Greenbased method was used and the relative quantitation of gene expression was determined using the comparative CT $\left({ }^{\Delta \Delta} \mathrm{CT}\right)$ method and normalized to the $\beta$-actin gene.

The PCR primer sequences used are given in the Supplementary Table S1. The mean \pm SD of three independent experiments is shown.

Cell proliferation assay, migration and invasion assay, and plate-colony formation assay

Details of the cell proliferation assay were described previously ${ }^{29}$. For plate-colony formation assay, cells $(2 \times$ $10^{3} / \mathrm{ml} /$ well) were seeded into 6 -well plates and cultured in RPMI-1640 medium supplemented with 10\% FBS. Colonies were fixed with methanol and stained with viola crystalline, then scored using a microscope and Image J software (1.47V, NIH, USA).

The migration assay was described previously ${ }^{24}$. Cells $\left(5 \times 10^{5}\right)$ were seeded onto the upper chamber in $200 \mu \mathrm{l}$ of serum-free medium; the lower compartment was filled with $0.6 \mathrm{ml}$ of DMEM media supplemented with $10 \%$ of FBS. After $24 \mathrm{~h}$ incubation, migrated cells on the lower surface of the filter were fixed and stained using propidium iodide. Cells on the upper side were removed using a rubber scraper. Fluorescent images were obtained. The reported data represent the counts of migrated cells. Experiments were performed in triplicates.

For the plate-colony formation assay, cells $\left(1 \times 10^{3} / \mathrm{ml} /\right.$ well) were seeded into 6-well plates and cultured in RPMI-1640 medium supplemented with 10\% FBS. Colonies were fixed with methanol, stained with viola crystallina and scored using a microscope and ImageJ software $(1.47 \mathrm{~V}, \mathrm{NIH}, \mathrm{USA})$. Surviving colonies were counted and the surviving fraction (SF) was calculated using the formula $\mathrm{SF}=$ treatment colony numbers/control colony numbers.

\section{Soft-agar colony forming assay}

To assess anchorage-independent growth, soft-agar colony forming assay was performed using 8000 cells per well. A layer of agar containing $3.0 \mathrm{ml}$ of $0.6 \%$ soft agar (BD Biosciences, USA) in (basement membrane extract) BME was poured into wells of a 6-well cell culture dish and allowed to set at room temperature for $30 \mathrm{~min}$. A second layer containing $1 \mathrm{ml} 0.35 \%$ soft agar in BME containing cells $(800$ cells $/ \mathrm{ml})$ was placed on the top of the first layer and allowed to set at room temperature for $30 \mathrm{~min}$. Cells were incubated in an incubator at $37^{\circ} \mathrm{C}$ for 14 days, and the number of colonies were counted, and the images were captured using an Olympus microscope.

\section{Oncosphere formation assay}

Cells were seeded on ultra-low attachment culture dishes (Corning, Corning, NY) in serum-free DMEM-F12 medium containing $50 \mu \mathrm{g} / \mathrm{ml}$ insulin (Sigma-Aldrich St. Louis, MO), 0.4\% Albumin Bovine Fraction V (SigmaAldrich St. Louis, MO), N-2 Plus Media Supplement (Life Technologies, Grand Island, NY), B-27 Supplement (Life Technologies, Grand Island, NY), 20 $\mathrm{g} / \mathrm{ml}$ EGF (PeproTech Rocky Hill, NJ), and $10 \mu \mathrm{g} / \mathrm{ml}$ basic FGF (PeproTech, Rocky Hill, NJ) to support the growth of undifferentiated oncospheres. Cells were incubated in a $\mathrm{CO}_{2}$ incubator for 1-2 weeks, and the numbers of oncosphere cells were counted under a microscope.

\section{Immunofluorescence assay and Operetta ${ }^{\circledR}$ High Content Screening and High Content Analysis}

Details of the immunofluorescence assays were described previously ${ }^{24,29}$, cells were cultured and fixed in $4 \%$ paraformaldehyde for $30 \mathrm{~min}$. To identify the potential presence of AhR and IKK $\alpha$, cells were incubated with an anti-AhR antibody (Sigma) and an anti-IKK $\alpha$ antibody (Active motif) and then with fluorescein isothiocyanate (FITC)-conjugated anti-IgG (Santa Cruz) and Cy3conjugated anti-IgG (Sigma). To visualize the nuclei, the cells were stained with Hoechst (1:1000). Fluorescent images were observed and analyzed with a laser scanning confocal microscope (Bio-Rad MRC-1024ES).

For Operetta ${ }^{\circledR}$ High Content Screening and High Content Analysis, cells were grown on the 96-well plate. After incubation overnight, the cells were washed with phosphate-buffered saline (PBS) and fixed with methanol for $10 \mathrm{~min}$ at $37^{\circ} \mathrm{C}$. The cells were incubated in PBS supplemented with $1 \%$ BSA for $1 \mathrm{~h}$ at room temperature. The cells were incubated overnight with E-cadherin mouse monoclonal antibody (1:100 dilution, abcam 1416) or Vimentin rabbit monoclonal antibody (1:200 dilution, CST 5741s) at $4{ }^{\circ} \mathrm{C}$. The cells were washed three times in PBS and then stained with Alexa Fluor 594 Goat Anti-Mouse IgG or Alexa Fluor 594 Goat Anti-Rabbit IgG (1:4000 dilution, Invitrogen) for $1 \mathrm{~h}$ at room temperature. After incubation with DAPI to stain the nuclei, the cells were imaged on the Operetta ${ }^{\circledR}$ High Content Imaging System and analyzed using Harmony ${ }^{\circledR}$ High Content Image Analysis Software (USA).

\section{ALDEFLUOR assay, flow cytometry, and cell sorting}

Cells were stained directly using different antibodies (including CD338-PerCP-Cy ${ }^{\mathrm{m}} 5.5, \quad$ CD326-BV510) according to the manufacturer's instructions. Labeled cells were detected using a FACSCalibur (BD Immunocytometry Systems, CA, USA) and analyzed with Flowjo software. IgG isotype controls corresponding to each directly conjugated fluorophore were utilized to identify, quantify, and positively select desired cell 
populations. Debris and cell clusters were excluded during side-scatter and forward-scatter analyses. For cell sorting, cells were stained and followed by sorting with FACS Aria III (BD Immunocytometry Systems, CA, USA). The separated populations were used for RNA and animal experiments.

The ALDEFLUOR kit (StemCell Technologies Inc.) was used as described previously ${ }^{64}$ and with the guidance of Dr. Suling Liu (Fudan University, Shanghai, China).

\section{Chromatin immunoprecipitation (ChIP) assays}

ChIP assays were essentially performed as previously described ${ }^{24,29}$ with modifications: $5 \times 10^{6}$ cells were fixed with formaldehyde ( $1 \%$ final volume concentration, Sigma), $10 \mathrm{~min}$ at room temperature. Fixation was stopped by the addition of $1 / 10$ volume $1.25 \mathrm{M}$ glycine and incubated for $5 \mathrm{~min}$ at room temperature. The sonication step was performed in a Qsonica sonicator $(5 \mathrm{~min}, 20 \mathrm{~s}$ on, $20 \mathrm{~s}$ off), and $200 \mu \mathrm{g}$ of protein-chromatin complex was used for each immunoprecipitation. Antibody-protein complex was captured with preblocked dynabeads protein G (Invitrogen). ChIP DNA was analyzed by qPCR with SYBR Green (Biorad) in ABI-7500 (Applied Biosystems) using the primers specified in Supplemental Table S2. The antibodies used are as indicated in Supplemental Table S3.

\section{Nude mice and study approval}

A xenograft tumor formation was essentially performed as previously described ${ }^{26}$, whereas the Hunan SJA laboratory Animal Co. Ltd. (http://www.hnsja.com) provided the mice. SCID mice were injected with indicated cells $\left(1 \times 10^{6}\right.$ cells/mouse or as indicated) via mammary fat pad or tail vein (6 mice/group or as indicated). Mice with A549-IR cells or indicated cells were imaged from dorsal and ventral views once per week. All mice (6 of 6) injected with cells via the tail developed the expected lung metastatic lesions within 8 weeks. Visible lung metastatic nodules were examined macroscopically or detected in paraffin-embedded sections stained with H\&E. Data were analyzed using Student's $t$-test; a $p$ value $<0.05$ was considered significant.

All procedures for animal study were approved by the Institutional Animal Care and Use Committee of the Central South University of Xiangya School of Medicine and conform to the legal mandates and federal guidelines for the care and maintenance of laboratory animals.

NCI-Frederick is accredited by AAALAC International and follows the Public Health Service Policy for the Care and Use of Laboratory Animals. Animal care was provided in accordance with the procedures outlined in the "Guide for Care and Use of Laboratory Animals (National Research Council; 1996; National Academy Press; Washington, DC).

\section{Immunohistochemistry (IHC) analysis and in situ hybridization of tumor biopsies}

NPC biopsies, validated by pathologist Dr. Desheng Xiao (Xiangya Hospital), were obtained from the Department of Pathology of Xiangya Hospital. The NPC tissue array was purchased from Pantomics (Richmond, CA, USA). IHC analysis of paraffin sections from NPC patient or xenograft samples was performed as described previously ${ }^{26,65}$. In situ hybridization was performed using the EBV-encoded RNA (EBER) HRP conjugated probe and $D A B$ as substrate from the ISH kit (Life technologies), according to the manufacturer's instructions.

\section{Statistics}

The experiments were repeated at least three times except the nude mice experiments. Results are expressed as mean \pm SD or SEM as indicated. All statistical analyses were performed using Prism 6.0 GraphPad Software. Significant differences between two groups were compared using the Student's $t$-test, and comparisons among more than two groups were performed using analysis of variance (ANOVA). The correlation analysis was conducted using Pearson's correlation coefficient. A $p$ value less than 0.05 was considered statistically significant.

Additional information can be found in the Supplementary Materials and Methods section.

\section{Acknowledgements}

The content of this publication does not necessarily reflect the views or policies of the Department of Health and Human Services, nor does it mention of trade names, commercial products, or organizations imply endorsement by the US Government. We thank Dr. Suling Liu (Fudan University, Shanghai, China) very much for the kind support of ALDEFLUOR assay. This work was supported by the National Basic Research Program of China (2015CB553903 (Y. T.)); the National Natural Science Foundation of China (81672787 and 81372427 (Y.T.), 81672991 and 81271763 (S.L.), 81302354 (Y.S.), 81422051 and 81472593 (Y.C.)); and the Fundamental Research Funds for the Central Universities (2013ZZTS074 (B.Y.)). This project has been funded in part with Federal funds from the Frederick National Laboratory for Cancer Research, National Institutes of Health, under contract HHSN261200800001E (K.M.).

\section{Author details}

${ }^{1}$ Institute of Medical Sciences, Xiangya Hospital, Central South University, 87 Xiangya Road, Changsha 410008 Hunan, China. ${ }^{2}$ Key Laboratory of

Carcinogenesis and Cancer Invasion, Ministry of Education, Xiangya Hospital, Central South University, 87 Xiangya Road, Changsha 410008 Hunan, China. ${ }^{3}$ Cancer Research Institute, Central South University, 110 Xiangya Road, Changsha 410078 Hunan, China. ${ }^{4}$ Department of Thoracic Surgery, Second Xiangya Hospital, Central South University, Changsha, China. ${ }^{5}$ Department of Pathology, Xiangya Hospital, Central South University, Changsha 410008, Hunan, China. ${ }^{6}$ National and Local Joint Engineering Laboratory of Highthroughput Molecular Diagnosis Technology, Translational Medicine Institute, the First People's Hospital of Chenzhou, University of South China, 102 Luojiajing Road, Chenzhou 423000 Hunan, China. ${ }^{7}$ Department of Pathophysiology, Changzhi Medical College, Changzhi, Shanxi, China.

${ }^{8}$ Department of Neurosugery, Xiangya Hospital, Central South University, 87 Xiangya Road, Changsha 410078 Hunan, China. ${ }^{9}$ Shanghai Institute of Material Medica, Chinese Academy of Sciences (CAS), 555 Zu Chongzhi Road, Zhangjiang Hi-Tech Park, 201203 Shanghai, China. ${ }^{10}$ Department of Pharmacology, School of Pharmaceutical Sciences, Central South University, Changsha 410078 Hunan, China. ${ }^{11}$ Cipher Gene (Beijing) Co. Ltd., 100089 Beijing, China. ${ }^{12}$ Mouse Cancer Genetics Program, National Cancer Institute, 
Basic Science Program, Leidos Biomedical Research, Inc., Frederick National Laboratory for Cancer Research, Frederick, MD 21702, USA

\section{Conflict of interest}

The authors declare that they have no conflict of interest. This manuscript has been read and approved by all the authors, and not submitted or under consideration for publication elsewhere.

\section{Publisher's note}

Springer Nature remains neutral with regard to jurisdictional claims in published maps and institutional affiliations.

Supplementary Information accompanies this paper at (https://doi.org/ 10.1038/s41419-018-0542-9).

\section{Received: 19 February 2018 Revised: 4 March 2018 Accepted: 27 March} 2018

Published online: 30 April 2018

\section{References}

1. Stejskalova, L., Dvorak, Z. \& Pavek, P. Endogenous and exogenous ligands of aryl hydrocarbon receptor: current state of art. Curr. Drug Metab. 12, 198-212 (2011).

2. Opitz, C. A. et al. An endogenous tumour-promoting ligand of the human ary hydrocarbon receptor. Nature 478, 197-203 (2011).

3. Bessede, A. et al. Aryl hydrocarbon receptor control of a disease tolerance defence pathway. Nature 511, 184-190 (2014).

4. Schiering, $C$. et al. Feedback control of AHR signalling regulates intestinal immunity. Nature 542, 242-245 (2017).

5. Villa, M. et al. Aryl hydrocarbon receptor is required for optimal B-cell proliferation. EMBO J. 36, 116-128 (2017)

6. Murray, I. A., Patterson, A. D. \& Perdew, G. H. Aryl hydrocarbon receptor ligands in cancer: friend and foe. Nat. Rev. Cancer 14, 801-814 (2014).

7. Cheng, J. et al. Tryptophan derivatives regulate the transcription of Oct4 in stem-like cancer cells. Nat. Commun. 6, 7209 (2015).

8. Hayden, M. S. \& Ghosh, S. Shared principles in NF-kappaB signaling. Cell 132 344-362 (2008)

9. Perkins, N. D. Integrating cell-signalling pathways with NF-kappaB and IKK function. Nat. Rev. Mol. Cell Biol. 8, 49-62 (2007).

10. Karin, M. \& Greten, F. R. NF-kappaB: linking inflammation and immunity to cancer development and progression. Nat. Rev. Immunol. 5, 749-759 (2005).

11. Senftleben, U. et al. Activation by IKKalpha of a second, evolutionary conserved, NF-kappa B signaling pathway. Science 293, 1495-1499 (2001).

12. Vallabhapurapu, S. \& Karin, M. Regulation and function of NF-kappaB transcription factors in the immune system. Annu. Rev. Immunol. 27, 693-733 (2009).

13. Xiao, D. et al. Opposed expression of IKKalpha: loss in keratinizing carcinomas and gain in non-keratinizing carcinomas. Oncotarget 6, 25499-25505 (2015).

14. Baumann, M., Krause, M. \& Hill, R. Exploring the role of cancer stem cells in radioresistance. Nat. Rev. Cancer 8, 545-554 (2008).

15. Skvortsova, I., Debbage, P., Kumar, V. \& Skvortsov, S. Radiation resistance: cancer stem cells (CSCS) and their enigmatic pro-survival signaling. Semin. Cancer Biol. 35, 39-44 (2015)

16. Roche, K. C. et al. SOX9 maintains reserve stem cells and preserves radioresistance in mouse small intestine. Gastroenterology 149, 1553-1563 e1510 (2015).

17. Kurth, I. et al. Cancer stem cell related markers of radioresistance in head and neck squamous cell carcinoma. Oncotarget 6, 34494-34509 (2015).

18. Cojoc, M. et al. Aldehyde dehydrogenase is regulated by beta-catenin/TCF and promotes radioresistance in prostate cancer progenitor cells. Cancer Res. 75, 1482-1494 (2015)

19. Shi, Y. et al. Nuclear EGFR-PKM2 axis induces cancer stem cell-like characteristics in irradiation-resistant cells. Cancer Lett. 422, 81-93 (2018).

20. Plaks, V., Kong, N. \& Werb, Z. The cancer stem cell niche: how essential is the niche in regulating stemness of tumor cells? Cell Stem Cell $\mathbf{1 6}$ 225-238 (2015).
21. Puga, A., Ma, C. \& Marlowe, J. L. The aryl hydrocarbon receptor cross-talks with multiple signal transduction pathways. Biochem. Pharmacol. 77, 713-722 (2009).

22. McCarthy, N. Therapeutics: targeting an oncometabolite. Nat. Rev. Cancer 13, 383 (2013).

23. Ing-Simmons, E. \& Merkenschlager, M. Oncometabolite tinkers with genome folding, boosting oncogene expression. Trends Mol. Med. 22, 185-187 (2016).

24. He, X. et al. Chromatin remodeling factor LSH drives cancer progression by suppressing the activity of fumarate hydratase. Cancer Res. 76, 5743-5755 (2016).

25. Jia, J. et al. LGR5 expression is controled by IKKalpha in basal cell carcinoma through activating STAT3 signaling pathway. Oncotarget 7, 27280-27294 (2016).

26. Jiang, Y. et al. Repression of Hox genes by LMP1 in nasopharyngeal carcinoma and modulation of glycolytic pathway genes by HoxC8. Oncogene 34 6079-6091 (2015).

27. $\mathrm{Xu}, \mathrm{J}$. et al. Tyrosylprotein sulfotransferase-1 and tyrosine sulfation of chemokine receptor 4 are induced by Epstein-Barr virus encoded latent membrane protein 1 and associated with the metastatic potential of human nasopharyngeal carcinoma. PLoS ONE 8, e56114 (2013).

28. Wang, $R$. et al. The ratio of FoxA1 to FoxA2 in lung adenocarcinoma is regulated by LncRNA HOTAIR and chromatin remodeling factor LSH. Sci. Rep. 5, 17826 (2015)

29. Shi, Y. et al. Nuclear epidermal growth factor receptor interacts with transcriptional intermediary factor 2 to activate cyclin D1 gene expression triggered by the oncoprotein latent membrane protein 1. Carcinogenesis 33, 1468-1478 (2012)

30. Mandl, M., Lieberum, M., Dunst, J. \& Depping, R. The expression level of the transcription factor Aryl hydrocarbon receptor nuclear translocator (ARNT) determines cellular survival after radiation treatment. Radiat. Oncol. 10, 229 (2015).

31. Prud'homme, G. J. et al. Breast cancer stem-like cells are inhibited by a nontoxic aryl hydrocarbon receptor agonist. PLOS ONE 5 e13831 (2010).

32. Stanford, E. A. et al. The role of the aryl hydrocarbon receptor in the development of cells with the molecular and functional characteristics of cancer stem-like cells. BMC Biol. 14, 20 (2016).

33. Al-Dhfyan, A., Alhoshani, A. \& Korashy, H. M. Aryl hydrocarbon receptor/ cytochrome P450 1A1 pathway mediates breast cancer stem cells expansion through PTEN inhibition and beta-Catenin and Akt activation. Mol. Cancer 16, 14 (2017).

34. Stanford, E. A. et al. Role for the aryl hydrocarbon receptor and diverse ligands in oral squamous cell carcinoma migration and tumorigenesis. Mol. Cancer Res. 14, 696-706 (2016).

35. Tan, K. P. et al. Aryl hydrocarbon receptor is a transcriptional activator of the human breast cancer resistance protein (BCRP/ABCG2). Mol. Pharmacol. 78, 175-185 (2010).

36. Chen, W. S. et al. Nuclear IKKalpha mediates microRNA-7/-103/107/21 inductions to downregulate maspin expression in response to $\mathrm{HBx}$ overexpression. Oncotarget 7, 56309-56323 (2016).

37. Kim, S. H. et al. Serine/threonine kinase MLK4 determines mesenchymal identity in glioma stem cells in an NF-kappaB-dependent manner. Cancer Cell 29, 201-213 (2016).

38. Cao, Y., Luo, J. L. \& Karin, M. IkappaB kinase alpha kinase activity is required for self-renewal of ErbB2/Her2-transformed mammary tumor-initiating cells. Proc. Natl Acad. Sci. USA 104, 15852-15857 (2007).

39. Luo, J. L. et al. Nuclear cytokine-activated IKKalpha controls prostate cancer metastasis by repressing Maspin. Nature 446, 690-694 (2007).

40. Tan, W. et al. Tumour-infiltrating regulatory $T$ cells stimulate mammary cancer metastasis through RANKL-RANK signalling. Nature 470, 548-553 (2011).

41. Zhang, W. et al. A NIK-IKKalpha module expands ErbB2-induced tumor-initiating cells by stimulating nuclear export of p27/Kip1. Cancer Cell 23, 647-659 (2013).

42. Goktuna, S. I. et al. IKKalpha promotes intestinal tumorigenesis by limiting recruitment of M1-like polarized myeloid cells. Cell Rep. 7, 1914-1925 (2014).

43. Liu, B. et al. IKKalpha is required to maintain skin homeostasis and prevent skin cancer. Cancer Cell 14, 212-225 (2008).

44. Xiao, Z. et al. The pivotal role of IKKalpha in the development of spontaneous lung squamous cell carcinomas. Cancer Cell 23, 527-540 (2013).

45. Thiery, J. P., Acloque, H., Huang, R. Y. \& Nieto, M. A. Epithelial-mesenchymal transitions in development and disease. Cell 139, 871-890 (2009). 
46. Mani, S. A. et al. The epithelial-mesenchymal transition generates cells with properties of stem cells. Cell 133, 704-715 (2008).

47. Lamouille, S., Xu, J. \& Derynck, R. Molecular mechanisms of epithelialmesenchymal transition. Nat. Rev. Mol. Cell Biol. 15, 178-196 (2014).

48. David, C. J. et al. TGF-beta tumor suppression through a lethal EMT. Cell 164 1015-1030 (2016)

49. Zheng, $X$. et al. Epithelial-to-mesenchymal transition is dispensable for metastasis but induces chemoresistance in pancreatic cancer. Nature $\mathbf{5 2 7}$ 525-530 (2015)

50. Fischer, K. R. et al. Epithelial-to-mesenchymal transition is not required for lung metastasis but contributes to chemoresistance. Nature $\mathbf{5 2 7}$ 472-476 (2015).

51. Celia-Terrassa, T. \& Kang, Y. Distinctive properties of metastasis-initiating cells. Genes Dev. 30, 892-908 (2016).

52. Liu, S. et al. Breast cancer stem cells transition between epithelial and mesenchymal states reflective of their normal counterparts. Stem Cell Reports 2, 78-91 (2014).

53. Yu, M. et al. Circulating breast tumor cells exhibit dynamic changes in epithelial and mesenchymal composition. Science 339, 580-584 (2013).

54. Nieto, M. A., Huang, R. Y., Jackson, R. A. \& Thiery, J. P. Emt: 2016. Cell 166, 21-45 (2016).

55. Korkaya, H., Liu, S. \& Wicha, M. S. Breast cancer stem cells, cytokine networks, and the tumor microenvironment. J. Clin. Invest. 121, 3804-3809 (2011).
56. Todaro, M. et al. CD44v6 is a marker of constitutive and reprogrammed cancer stem cells driving colon cancer metastasis. Cell Stem Cell 14, 342-356 (2014).

57. Pavlova, N. N. \& Thompson, C. B. The emerging hallmarks of cancer metabolism. Cell Metab. 23, 27-47 (2016).

58. Hirayama, A. et al. Quantitative metabolome profiling of colon and stomach cancer microenvironment by capillary electrophoresis time-of-flight mass spectrometry. Cancer Res. 69, 4918-4925 (2009).

59. Yang, M., Soga, T. \& Pollard, P. J. Oncometabolites: linking altered metabolism with cancer. J. Clin. Invest. 123, 3652-3658 (2013).

60. Ho, P. C. et al. Phosphoenolpyruvate is a metabolic checkpoint of anti-tumor T cell responses. Cell 162, 1217-1228 (2015).

61. $\mathrm{Xu}, \mathrm{W}$. et al. Oncometabolite 2-hydroxyglutarate is a competitive inhibitor of alpha-ketoglutarate-dependent dioxygenases. Cancer Cell 19, 17-30 (2011).

62. Turcan, S. et al. IDH1 mutation is sufficient to establish the glioma hypermethylator phenotype. Nature 483, 479-483 (2012).

63. You, S. et al. Disruption of STAT3 by niclosamide reverses radioresistance of human lung cancer. Mol. Cancer Ther. 13, 606-616 (2014).

64. Ginestier, $\mathrm{C}$. et al. ALDH1 is a marker of normal and malignant human mammary stem cells and a predictor of poor clinical outcome. Cell Stem Cell $\mathbf{1}$ 555-567 (2007)

65. Xiao, D. et al. Chromatin remodeling factor LSH is upregulated by the LRP6GSK3beta-E2F1 axis linking reversely with survival in gliomas. Theranostics 7, 132-143 (2017). 